\title{
Summation over Feynman Histories in Polar Coordinates
}

\author{
D. Peak and A. Inomata \\ Department of Physics, State University of New York, Albany, New York
}

(Received 17 April 1968)

\begin{abstract}
Use of polar coordinates is examined in performing summation over all Feynman histories. Several relationships for the Lagrangian path integral and the Hamiltonian path integral are derived in the central-force problem. Applications are made for a harmonic oscillator, a charged particle in a uniform magnetic field, a particle in an inverse-square potential, and a rigid rotator. Transformations from Cartesian to polar coordinates in path integrals are rather different from those in ordinary calculus and this complicates evaluation of path integrals in polars. However, it is observed that for systems of central symmetry use of polars is often advantageous over Cartesians.
\end{abstract}

\section{INTRODUCTION}

Of fundamental importance to quantum mechanics is the Schrödinger equation

$$
-i \partial_{t} \psi(\mathbf{r}, t)=H \psi(\mathbf{r}, t)
$$

containing $H$, the Hamiltonian of the system, as a differential operator. This differential equation can be replaced by an integral equation

$$
\dot{\psi}\left(\mathbf{r}^{\prime \prime}, \tau\right)=\int K\left(\mathbf{r}^{\prime \prime}, \mathbf{r}^{\prime} ; \tau\right) \psi\left(\mathbf{r}^{\prime}, 0\right) d \mathbf{r}^{\prime},
$$

if the initial condition $\psi\left(\mathbf{r}^{\prime \prime}, 0\right)=\psi\left(\mathbf{r}^{\prime}, 0\right)$ is satisfied. The kernel of Eq. (2) corresponds to the propagator of the wavefunction $\psi(\mathbf{r}, t)$ from the point $\mathbf{r}^{\prime}$ to $\mathbf{r}^{\prime \prime}$ in time $\tau$.

In Feynman's Lagrangian formulation, ${ }^{1}$ it is asserted that the kernel is given by a path integral

$$
K\left(\mathbf{r}^{\prime \prime}, \mathbf{r}^{\prime} ; \tau\right)=\int \exp \left[i S\left(\mathbf{r}^{\prime \prime}, \mathbf{r}^{\prime}\right)\right] \operatorname{Dr}(t)
$$

Here, integrations are over all possible paths, or histories, starting at $\mathbf{r}^{\prime}=\mathbf{r}(0)$ and terminating at $\mathbf{r}^{\prime \prime}=\mathbf{r}(\tau)$. The function $S\left(\mathbf{r}^{\prime \prime}, \mathbf{r}^{\prime}\right)$ in the integrand is the classical action

$$
S\left(\mathbf{r}^{\prime \prime}, \mathbf{r}^{\prime}\right)=\int_{0}^{t} L(\dot{\mathbf{r}}, \mathbf{r}) d t,
$$

$L(\dot{\mathbf{r}}, \mathbf{r})$ being the Lagrangian of the system in question.

As an alternative approach to quantization, Feynman's formalism has attracted much attention. ${ }^{2}$ However, this approach is applicable only to a limited class of problems. ${ }^{3}$ Certainly any effort to extend it beyond its present limits would be worthwhile. In most applications available so far, calculations are done in Cartesian coordinates. It has been suggested that the integral over all paths may be performed in polar coordinates as well. ${ }^{4}$ It is the purpose of the

\footnotetext{
${ }^{1}$ R. P. Feynman, Rev. Mod. Phys. 20, 367 (1948).

2 S. G. Brush, Rev. Mod. Phys. 33, 79 (1961).

See, e.g., R. P. Feynman and A. R. Hibbs, Quantum Mechanics and Path Integrals (McGraw-Hill Book Co., Inc., New York, 1965).

4 S. F. Edwards and Y. V. Gulyaev, Proc. Roy. Soc. (London) A279, 229 (1964); S. Ozaki, Lectures at Kyushu University, 1955 (unpublished).
}

present paper to demonstrate the usefulness of polar coordinates in evaluating the path integral for specific particle systems. Indeed, it is observed that most solvable examples in Cartesians are equally well treated in polars. Use of polars seems of better advantage for certain systems of central symmetry, although the applications considered are all essentially of the harmonic-oscillator type.

In Sec. II, we derive several general expressions for the path integral in the central-force problem. The Hamiltonian path integral equivalent to Feynman's Lagrangian path integral is also discussed in polars. Section III is devoted to applications. The propagator of the harmonic oscillator is the first example, a limiting case of which includes the free particle. A slight modification of the procedure of computing the propagator for the harmonic oscillator in polars leads to the result of Sondheimer and Wilson for charged particles in a uniform magnetic field. ${ }^{5}$ The third example is the rigid rotator, for which the Hamiltonian path integral is utilized. The final calculation, concerned with a particle in an inverse-square potential, could hardly be completed in Cartesians but is found trivial in polars. In an appendix, derivations of the formulas used in the text are given. Throughout this paper we employ natural units, i.e., $\hbar=c=1$.

\section{THE CENTRAL-FORCE PROBLEM}

\section{The Lagrangian Path Integral}

It is customary to define the summation over Feynman histories (3) by ${ }^{3}$

$$
\begin{aligned}
& K\left(\mathbf{r}^{\prime \prime}, \mathbf{r}^{\prime} ; \tau\right) \\
& \quad=\lim _{N \rightarrow \infty} A_{N} \int \exp \left[i \sum_{j=1}^{N} S\left(\mathbf{r}_{j}, \mathbf{r}_{j-1}\right)\right] d \mathbf{r}_{1} d \mathbf{r}_{2} \cdots d \mathbf{r}_{N-\mathbf{1}},
\end{aligned}
$$

where $\mathbf{r}_{j}=\mathbf{r}\left(t_{j}\right), \mathbf{r}_{0}=\mathbf{r}^{\prime}, \mathbf{r}_{N}=\mathbf{r}^{\prime \prime}, t_{j}-t_{j-1}=\tau / N=$ $\epsilon$, and $A_{N}$ is the normalization factor in the $N$ th

${ }^{5}$ E. H. Sondheimer and A. H. Wilson, Proc. Roy. Soc. (London), A210, 173 (1951). For derivation by the path-integral method, see M. L. Glasser, Phys. Rev. 113, B831 (1964); A. Inomata, Benét Laboratories, U.S. Army, Technical Report WVT-6718, 1967. 
approximation. The partial action in a small time interval $\Delta t_{j}=t_{j}-t_{j-1}$ may be approximated by

$$
S\left(\mathbf{r}_{j}, \mathbf{r}_{j-1}\right) \approx \epsilon L\left(\Delta \mathbf{r}_{j} / \epsilon, \mathbf{r}_{j}\right),
$$

where $\Delta \mathbf{r}_{j}=\mathbf{r}_{j}-\mathbf{r}_{j-1}$ and $\Delta t_{j}=\epsilon$. This approximation reflects the situation that the important contributions to the path integral are only from the paths close to the classical one.

In polar coordinates, the squared distance between two points $\mathbf{r}_{j}\left(r_{j}, \theta_{j}, \phi_{j}\right)$ and $\mathbf{r}_{j-1}\left(r_{j-1}, \theta_{j-1}, \phi_{j-1}\right)$ is

where

$$
\left(\Delta \mathbf{r}_{j}\right)^{2}=r_{j}^{2}+r_{j-1}^{2}-2 r_{j} r_{j-1} \cos \Theta_{j}
$$

$$
\begin{aligned}
\cos \Theta_{j}=\cos \theta_{j} & \cos \theta_{j-1} \\
& +\sin \theta_{j} \sin \theta_{j-1} \cos \left(\phi_{j}-\phi_{j-1}\right) .
\end{aligned}
$$

For a particle of mass $m$ in a central potential, the partial action is given by

$$
\begin{aligned}
& S\left(\mathbf{r}_{j}, \mathbf{r}_{j-1}\right) \\
& \quad=\frac{1}{2} m\left(r_{j}^{2}+r_{j-1}^{2}\right) / \epsilon-(m / \epsilon) r_{j} r_{j-1} \cos \Theta_{j}-\epsilon V\left(r_{j}\right) .
\end{aligned}
$$

If use is made of the expansion formula

$$
\exp (u \cos \Theta)=\left(\frac{\pi}{2 u}\right)^{\frac{1}{2}} \sum_{l=0}^{\infty}(2 l+1) P_{l}(\cos \Theta) I_{l+\frac{1}{2}}(u)
$$

in terms of $P_{l}(\cos \Theta)$, the Legendre function, and $I_{l+\frac{1}{2}}(u)$, the modified Bessel function, the integrand of Eq. (5) can be written as

$$
\begin{aligned}
\exp & {\left[i \sum_{j=1}^{N} S\left(\mathbf{r}_{j}, \mathbf{r}_{j-1}\right)\right] } \\
& =\prod_{j=1}^{N}\left[\sum_{l_{j}=0}^{\infty}\left(2 l_{j}+1\right) P_{l_{j}}\left(\cos \Theta_{j}\right) R_{l_{j}}\left(r_{j}, r_{j-1}\right)\right],
\end{aligned}
$$

where

$$
\begin{aligned}
& R_{l}\left(r_{j}, r_{j-1}\right)=\left\{\frac{i \pi \epsilon}{2 m r_{j} r_{j-1}}\right\}^{\frac{1}{2}} \\
& \quad \times \exp \left[\frac{i m}{2 \epsilon}\left(r_{j}^{2}+r_{j-1}^{2}\right)-i \epsilon V\left(r_{j}\right)\right] I_{l+\frac{1}{2}}\left(\frac{m}{i \epsilon} r_{j} r_{j-1}\right) .
\end{aligned}
$$

After interchanging multiplications and summations, the right-hand side of Eq. (11) becomes

$$
\sum_{l_{1} l_{2} \cdots l_{N}}\left\{\prod_{j=1}^{N}\left[\left(2 l_{j}+1\right) P_{l_{j}}\left(\cos \Theta_{j}\right) R_{l_{j}}\left(r_{j}, r_{j-1}\right)\right]\right\} \text {. }
$$

Substitution of this result into Eq. (5) yields

$$
\begin{array}{r}
K\left(\mathbf{r}^{\prime \prime}, \mathbf{r}^{\prime} ; \tau\right)=\lim _{N \rightarrow \infty} A_{N} \sum_{l_{1} l_{2} \cdots l_{N}} \\
\times \int \prod_{j=1}^{N}\left\{\left(2 l_{j}+1\right) P_{l_{j}}\left(\cos \Theta_{j}\right) R_{l_{j}}\left(r_{j}, r_{j-1}\right)\right\} \\
\times \prod^{N-1}\left(r^{2} \sin \theta d r d \theta d \phi\right) .
\end{array}
$$

Here,

$$
\prod^{N-1}\left(r^{2} \sin \theta d r d \theta d \phi\right)=\prod_{j=1}^{N-1} r_{j}^{2} \sin \theta_{j} d r_{j} d \theta_{j} d \phi_{j} ;
$$

this convention will be adapted hereafter. The angular integrations in Eq. (13) can easily be carried out. First, expand $P_{l}(\cos \Theta)$ in terms of the spherical harmonics

$$
P_{l}\left(\cos \Theta_{j}\right)=\frac{4 \pi}{2 l+1} \sum_{n=-l}^{l} Y_{l}^{n *}\left(\theta_{j}, \phi_{j}\right) Y_{l}^{n}\left(\theta_{j-1}, \phi_{j-1}\right) .
$$

Then use the orthogonality relation

$$
\iint Y_{l}^{n *}(\theta, \phi) Y_{l^{\prime}}^{n^{\prime}}(\theta, \phi) \sin \theta d \theta d \phi=\delta_{l l^{\prime}} \delta_{n n^{\prime}}
$$

to obtain

$$
\begin{aligned}
\iint \prod_{j=1}^{N} & \left\{\left(2 l_{j}+1\right) P_{l_{j}}\left(\cos \Theta_{j}\right)\right\} \prod^{N-1}(\sin \theta d \theta d \phi) \\
& =(4 \pi)^{N} \delta_{l l_{N}} \prod_{j=1}^{N-1} \delta_{l_{j+1} l_{N}} \sum_{n=-l}^{l} Y_{l}^{n^{*}}\left(\theta^{\prime \prime}, \phi^{\prime \prime}\right) Y_{l}^{n}\left(\theta^{\prime}, \phi^{\prime}\right) .
\end{aligned}
$$

As a result, for each quantum number $l$, the radial and angular contributions to the propagator are separable; that is,

$$
\begin{aligned}
& K\left(r^{\prime \prime}, \theta^{\prime \prime}, \phi^{\prime \prime} ; r^{\prime}, \theta^{\prime}, \phi^{\prime} ; \tau\right) \\
& \quad=\sum_{l=0}^{\infty} \sum_{n=-l}^{l} K_{l}\left(r^{\prime \prime}, r^{\prime} ; \tau\right) Y_{l}^{n^{*}}\left(\theta^{\prime \prime}, \phi^{\prime \prime}\right) Y_{l}^{n}\left(\theta^{\prime}, \phi^{\prime}\right),
\end{aligned}
$$

with the radial propagator of the $l$ wave

$$
\begin{aligned}
& K_{l}\left(r^{\prime \prime}, r^{\prime} ; \tau\right) \\
& \quad=\lim _{N \rightarrow \infty}(4 \pi)^{N} A_{N} \int \prod_{j=1}^{N}\left\{R_{l}\left(r_{j}, r_{j-1}\right)\right\} \prod^{N-1}\left(r^{2} d r\right)
\end{aligned}
$$

remaining to be evaluated, contingent on specification of the potential. The normalization factor, so chosen that the total propagator (17) may be unitary, is

$$
A_{N}=(2 \pi i \epsilon / m)^{-\frac{3}{2} N} \text {. }
$$

\section{The Hamiltonian Path Integral}

It has been shown ${ }^{6}$ that in Cartesian coordinates

$$
\begin{aligned}
& A_{N} \int \exp \left[i \int L d t\right] \prod^{N-1}(d \mathbf{r}) \\
& =(2 \pi)^{-3 N} \iint \exp \left[i \int(\mathbf{p} \cdot \dot{\mathbf{r}}-H) d t\right] \prod^{N}(d \mathbf{p}) \prod^{N-1}(d) \mathbf{r},
\end{aligned}
$$

where $\mathbf{p}$ is the momentum conjugate to $\mathbf{r}$. This implies that the Hamiltonian path integral in phase space is

\footnotetext{
${ }^{6}$ H. Davies, Proc. Cambridge Phil. Soc. 59, 147 (1963); C. Garrod, Rev. Mod. Phys. 38, 483 (1966).
} 
identical to Feynman's Lagrangian path integral as far as particle systems described on the Cartesian basis are concerned. Since we are interested in the approximation (6), corrections higher than the first order in $\epsilon$ are unimportant. If the approximation

$$
\cos \delta \approx 1-\frac{1}{2} \delta^{2}
$$

is valid for angular changes $\delta$ in the time interval $\epsilon$, then one can express (20) in polars. However, the approximation (21) is not relevant, as Edwards and Gulyaev have pointed out. ${ }^{4}$ This may be compared with the situation that the simple procedure of replacing $p$ by $-i(\partial / \partial \mathrm{q})$ is not reliable in polars. The irrelevance arises from the fact that even if the changes in Cartesian variables are of the order of $\epsilon$, the corresponding changes in angular variables are not.

In order to take all contributions up to first order in $\epsilon$ into account, we utilize the asymptotic form of $I_{v}(u / \epsilon)$ for small $\epsilon$,

$$
I_{\nu}\left(\frac{u}{\epsilon}\right) \sim\left(\frac{2 \pi u}{\epsilon}\right)^{-\frac{1}{2}} \exp \left[\frac{u}{\epsilon}-\frac{1}{2}\left(v^{2}-\frac{1}{4}\right) \frac{\epsilon}{u}+O\left(\epsilon^{2}\right)\right],
$$

and replace Eq. (10) by

$\exp \left[\frac{u}{\epsilon} \cos \delta\right] \approx \frac{\epsilon}{2 u} \sum_{v=-\infty}^{\infty} \exp \left[i \nu \delta+\frac{u}{\epsilon}-\frac{\left(v^{2}-\frac{1}{4}\right) \epsilon}{2 u}\right]$.

Use of this approximation formula and the identity $\frac{1}{2} m(\Delta r)^{2}=p \Delta r-\frac{1}{2} \epsilon p^{2} / m+\frac{1}{2} \epsilon(p-m \Delta r / \epsilon)^{2} / m$

enable us to derive

$$
\begin{aligned}
\exp & {\left[i S\left(\mathbf{r}_{j}, \mathbf{r}_{j-1}\right)-\frac{i \epsilon}{2 m}\left(p_{j}-\frac{m}{\epsilon} \Delta r_{j}\right)^{2}\right] } \\
= & \frac{i \epsilon}{2 \pi m r_{j} r_{j-1}}\left(\sin \theta_{j} \sin \theta_{j-1}\right)^{\frac{1}{2}} \\
& \times \sum_{\mu, \nu} \exp \left[i p_{j} \Delta r_{j}+i \mu \Delta \theta_{j}+i v \Delta \phi_{j}-\frac{i \epsilon\left(\mu^{2}-\frac{1}{4}\right)}{2 m r_{j} r_{j-1}}\right. \\
& \left.-\frac{i \epsilon\left(\nu^{2}-\frac{1}{4}\right)}{2 m r_{j} r_{j-1} \sin \theta_{j} \sin \theta_{j-1}}-i \epsilon V\left(r_{j}\right)\right]
\end{aligned}
$$

Integrating both sides of (25) over the entire range of $p_{j}$ and dividing by the constant factor resulting from the Fresnel integral on the left-hand side yield

$$
\begin{aligned}
& \exp \left[i S\left(\mathbf{r}_{j}, \mathbf{r}_{j-1}\right)\right] \\
&=\left(\frac{i \epsilon}{2 \pi m}\right)^{\frac{3}{2}}\left(r_{j} r_{j-1} \sin \theta_{j} \sin \theta_{j-1}\right)^{-\frac{1}{2}} \\
& \times \sum_{\mu, v} \int \exp \left[i p_{j} \Delta r_{j}+i \mu \Delta \theta_{j}+i v \Delta \phi_{j}-\frac{i \epsilon\left(\mu^{2}-\frac{1}{4}\right)}{2 m r_{j} r_{j-1}}\right. \\
&\left.-\frac{i \epsilon\left(\nu^{2}-\frac{1}{4}\right)}{2 m r_{j} r_{j-1} \sin \theta_{j} \sin \theta_{j-1}}-i \epsilon V\left(r_{j}\right)\right] d p_{j} .
\end{aligned}
$$

On substitution of (26), the path integral turns out to be of the Hamiltonian form, analogous to that in Eq. (20); namely, for $N$ large,

$$
\begin{aligned}
A_{N} \int \exp & {\left[i \int L d t\right] \prod^{N-1}\left(r^{2} \sin \theta d r d \theta d \phi\right) } \\
= & (2 \pi)^{-3 N} \int \exp \left[i \int\left(p \dot{r}+p_{\theta} \dot{\theta}+p_{\phi} \dot{\phi}-H\right) d t\right] \\
& \times \prod^{N}\left(d p d p_{\theta} d p_{\phi}\right) \prod^{N-1}(d r d \theta d \phi)
\end{aligned}
$$

where we have made formal replacements

$$
\begin{gathered}
\mu \rightarrow p_{\theta}, \quad \nu \rightarrow p_{\phi}, \\
p^{2}+\left(\mu^{2}-\frac{1}{4}\right) / r^{2}+\left(v^{2}-\frac{1}{4}\right) /\left(r^{2} \sin ^{2} \theta\right) \\
+2 m V(r) \rightarrow H,
\end{gathered}
$$

and

$$
\begin{aligned}
\sum_{\mu_{1} \mu_{2} \cdots \mu_{N}} \sum_{v_{1} \nu_{2} \cdots v_{N}} \rightarrow\left(r^{\prime 2} r^{\prime \prime 2} \sin \theta^{\prime} \sin \theta^{\prime \prime}\right)^{\frac{1}{2}} \\
\times \iint \prod^{N}\left(d p_{\theta} d p_{\phi}\right) .
\end{aligned}
$$

There is an essential feature of the representation in polars due to the premise that the system has rotational symmetry. Because of the periodicity associated with rotation, the angular momentum assumes only discrete values, so that the propagator may remain single-valued. In this regard, the replacements (28)(30) are literally formal. It may be worth noting that if the system is bounded by a finite cubic box, the representation in Cartesians also requires each component of the linear momentum to take discrete values. Then integrations over the momentum variables in Eq. (20) must be treated as summations over possible discrete values. The difference of symmetries assumed for the system is the main source of the difference between the features of the representations in Cartesians and in polars.

In fact, the angular motion is solely determined by the rotational symmetry, and much involved calculations are unnecessary. What remains to be determined is only the radial motion. It is therefore more practical to develop the Hamiltonian path integral for the radial propagator than to handle the formal expression (27). In the following, we shall derive the radial propagator for the $l$-wave in the Hamiltonian form. With the approximation formula (22), the radial function (12) is given by

$$
\begin{aligned}
& R_{l}\left(r_{j}, r_{j-1}\right) \\
& \quad=\frac{i \epsilon}{2 m r_{j} r_{j-1}} \exp \left[\frac{i m\left(\Delta r_{j}\right)^{2}}{2 \epsilon}-\frac{i \epsilon l(l+1)}{2 m r_{j} r_{j-1}}-i \epsilon V\left(r_{j}\right)\right] .
\end{aligned}
$$


In the same fashion as Eq. (23), we write the radial function as

$$
\begin{array}{r}
R_{l}\left(r_{j}, r_{j-1}\right)=\frac{1}{4}\left(\frac{2 \pi \epsilon i}{m}\right)^{\frac{1}{2}}\left(r_{j} r_{j-1}\right)^{-1} \int_{-\infty}^{\infty} \exp \left[i p_{j} \Delta r_{j}\right. \\
\left.-\frac{i \epsilon}{2 m} p_{j}^{2}-\frac{i \epsilon l(l+1)}{2 m r_{j} r_{j-1}}-i \epsilon V\left(r_{j}\right)\right] d p_{j} .
\end{array}
$$

Equation (31) shows that the radial propagator for the $l$ wave is

$$
\begin{aligned}
& K_{l}\left(r^{\prime \prime}, r^{\prime} ; \tau\right)=\left(r^{\prime} r^{\prime \prime}\right)^{-1} \lim _{N \rightarrow \infty}\left(\frac{m}{2 \pi i \epsilon}\right)^{\frac{1}{2} N} \\
& \quad \times \int \exp \left\{i \int\left[\frac{1}{2} m \dot{r}^{2}-\frac{l(l+1)}{r^{2}}-V(r)\right] d t\right\} \prod^{N-1}(d r),
\end{aligned}
$$

which coincides with the results of Ozaki and of Edwards and Gulyaev ${ }^{4}$ for $V(r)=0$. On the other hand, use of expression (32) leads to the Hamiltonian path integral

$$
\begin{aligned}
& K_{l}\left(r^{\prime \prime}, r^{\prime} ; \tau\right)=\left(r^{\prime} r^{\prime \prime}\right)^{-1} \lim _{N \rightarrow \infty}\left(\frac{m}{2 i \epsilon}\right)^{N} \\
& \quad \times \iint \exp \left[i \int\left(p \dot{r}-H_{l}\right) d t\right] \prod^{N}(d p) \prod^{N-1}(d r),
\end{aligned}
$$

where

$$
H_{l}=\frac{1}{2 m}\left[p^{2}+\frac{l(l+1)}{r^{2}}\right]+V(r) .
$$

\section{APPLICATIONS}

\section{The Harmonic Oscillator}

For the harmonic oscillator having spring constant $k=m \omega^{2}$, the Lagrangian is

$$
L=\frac{1}{2} m\left(\dot{\mathbf{r}}^{2}-\omega^{2} r^{2}\right)
$$

and, hence, the partial action in the time interval $\Delta t_{j}$ is given by

$$
\begin{aligned}
S\left(\mathbf{r}_{j}, \mathbf{r}_{j-1}\right)= & \frac{1}{2}(m / \epsilon)\left(r_{j}^{2}+r_{j-1}^{2}\right) \\
& -(m / \epsilon) r_{j} r_{j-1} \cos \Theta_{j}-\frac{1}{2} \epsilon m \omega^{2} r_{j}^{2} .
\end{aligned}
$$

The corresponding radial function reads

$$
\begin{aligned}
& R_{l}\left(r_{j}, r_{j-1}\right)= {\left[\frac{i \pi \epsilon}{2 m r_{j} r_{j-1}}\right]^{\frac{1}{2}} } \\
& \times \exp \left[\frac{i m}{2 \epsilon}\left(r_{j}^{2}+r_{j-1}^{2}\right)+\frac{1}{2} i \epsilon m \omega^{2} r_{j}^{2}\right] \\
& \times I_{l+\frac{1}{2}}\left(\frac{m}{i \epsilon} r_{j} r_{j-1}\right),
\end{aligned}
$$

with which the radial propagator of the $l$ wave can be put in the form

$$
\begin{aligned}
& K_{l}\left(r^{\prime \prime}, r^{\prime} ; \tau\right)=\left(r^{\prime} r^{\prime \prime}\right)^{-\frac{1}{2}} \lim _{N \rightarrow \infty}(-i \beta)^{N} \exp \left[\frac{1}{2} i \beta\left(r^{\prime 2}+r^{\prime 2}\right)\right] \\
& \quad \times \int \exp \left[i \alpha\left(r_{1}^{2}+r_{2}^{2}+\cdots+r_{N-1}^{2}\right)\right] \\
& \quad \times I_{l+\frac{1}{2}}\left(-i \beta r_{0} r_{1}\right) \cdots I_{l+\frac{1}{2}}\left(-i \beta r_{N-1} r_{N}\right) \prod^{N-1}(r d r),
\end{aligned}
$$

where

$$
\beta=m / \epsilon, \quad \alpha=\beta\left(1-\frac{1}{2} \omega^{2} \epsilon^{2}\right) .
$$

As is shown in Appendix A, the formula

$$
\begin{aligned}
& \int_{0}^{\infty} \exp \left(i \alpha r^{2}\right) I_{v}(-i a r) I_{v}(-i b r) r d r \\
& =\frac{i}{2 \alpha} \exp \left[\frac{-i\left(a^{2}+b^{2}\right)}{4 \alpha}\right] I_{v}\left(-i \frac{a b}{2 \alpha}\right)
\end{aligned}
$$

is valid for $\operatorname{Re}(y)>-1$ and $\operatorname{Re}(\alpha)>0$. Repeated use of the above formula yields

$$
\begin{gathered}
\int \exp \left[i \alpha\left(r_{1}^{2}+\cdots+r_{N-1}^{2}\right)\right] I_{v}\left(-i \beta r_{0} r_{1}\right) \cdots \\
I_{v}\left(-i \beta r_{N-1} r_{N}\right) \prod^{N-1}(r d r)=\prod_{j=1}^{N-1}\left(\frac{i}{2 \alpha_{j}}\right) \\
\times \exp \left\{-i\left[r^{\prime 2} \sum_{j=1}^{N-1} \frac{\beta_{j}^{2}}{4 \alpha_{j}}+r^{\prime 2} \frac{\beta^{2}}{\alpha_{N}}\right]\right\} I_{v}\left(-i \beta_{N} r_{0} r_{1}\right),
\end{gathered}
$$

where $\alpha_{j}$ and $\beta_{j}$ are coefficients to be determined by solving the following algebraic equations:

$$
\begin{aligned}
& \alpha_{1}=\alpha, \quad \alpha_{j+1}=\alpha-\frac{\beta^{2}}{4 \alpha_{j}}, \quad \text { for } j \geq 1, \\
& \beta_{1}=\beta, \quad \beta_{j+1}=\beta \prod_{k=1}^{j} \frac{\beta}{2 \alpha_{k}}, \quad \text { for } j \geq 1 .
\end{aligned}
$$

The multi-integral formula (42) enables us to complete the radial integrations in Eq. (39); i.e.,

$$
\begin{aligned}
& K_{l}\left(r^{\prime \prime}, r^{\prime} ; \tau\right)=-i\left(r^{\prime} r^{\prime \prime}\right)^{-\frac{1}{2}} \lim _{N \rightarrow \infty} a_{N} \\
& \quad \times \exp \left(i f_{N} r^{\prime 2}+i g_{N} r^{\prime 2}\right) I_{l+\frac{1}{2}}\left(-i a_{N} r^{\prime} r^{\prime \prime}\right) .
\end{aligned}
$$

Our problem reduces to determining the factors

$$
\begin{aligned}
& a_{N}=\prod_{j=1}^{N-1} \frac{\beta}{2 \alpha_{j}}, \\
& f_{N}=\frac{1}{2} \beta-\frac{1}{4} \sum_{j=1}^{N-1} \frac{\beta_{j}^{2}}{\alpha_{j}}, \\
& g_{N}=\frac{1}{2} \beta-\frac{1}{4} \frac{\beta^{2}}{\alpha_{N}} .
\end{aligned}
$$

As is seen in Appendix B, the coefficient $\alpha_{j}$ satisfying Eq. (43) can be given in terms of a polynomial so that 
the factors $a_{N}, f_{N}$, and $g_{N}$ defined above are expressible in series form. However, what we are interested in is the limiting value of each factor for $N \rightarrow \infty$. In Appendix $\mathrm{B}$, it is also shown that as $N$ tends to infinity

$$
\begin{aligned}
& a_{N} \rightarrow m \omega \csc (\omega \tau), \\
& f_{N} \rightarrow \frac{1}{2} m \omega \cot (\omega \tau), \\
& g_{N} \rightarrow \frac{1}{2} m \omega \cot (\omega \tau) .
\end{aligned}
$$

Therefore, the radial propagator becomes

$$
\begin{aligned}
& K_{l}\left(r^{\prime \prime}, r^{\prime} ; \tau\right)=-i\left(r^{\prime} r^{\prime \prime}\right)^{-\frac{1}{2}} m \omega \csc (\omega \tau) \\
& \times \exp \left[\frac{1}{2} i m \omega\left(r^{\prime 2}+r^{\prime \prime 2}\right) \cot (\omega \tau)\right] \\
& \times I_{l+\frac{1}{2}}\left[-i m \omega r^{\prime} r^{\prime \prime} \csc (\omega \tau)\right] .
\end{aligned}
$$

As a particular case, the propagator of the twodimensional oscillator can be obtained:

$$
\begin{aligned}
& K\left(r^{\prime \prime}, \phi^{\prime \prime} ; r^{\prime}, \phi^{\prime} ; \tau\right)=\frac{m \omega}{2 \pi i \sin (\omega \tau)} \exp \left\{\frac{i m \omega}{2 \sin (\omega \tau)}\right. \\
& \left.\quad \times\left[\left(r^{\prime 2}+r^{\prime \prime 2}\right) \cos (\omega \tau)-2 r^{\prime} r^{\prime \prime} \cos \left(\phi^{\prime \prime}-\phi^{\prime}\right)\right]\right\} .
\end{aligned}
$$

For the one-dimensional oscillator,

$$
\begin{aligned}
& K\left(r^{\prime \prime}, r^{\prime} ; \tau\right) \\
& \quad=\left(\frac{m \omega}{2 \pi i \sin (\omega \tau)}\right)^{\frac{1}{2}} \exp \left[\frac{1}{2} i m \omega\left(r^{\prime 2}+r^{\prime \prime 2}\right) \cot (\omega \tau)\right] .
\end{aligned}
$$

In the limit where $\omega$ vanishes, the propagator (52) reduces to that of a free particle in three dimensions. In the same limit, the propagator (54) leads to the onedimensional free-particle case

$$
K_{0}\left(r^{\prime \prime}, r^{\prime} ; \tau\right)=\left(\frac{m}{2 \pi i \tau}\right)^{\frac{1}{2}} \exp \left[\frac{i m}{2 \tau}\left(r^{\prime \prime}-r^{\prime}\right)^{2}\right]
$$

From Eqs. (33) and (52) follows the useful relation

$$
\begin{aligned}
\int & \exp \left\{i \int\left[\frac{1}{2} m \dot{r}^{2}-\frac{v^{2}-\frac{1}{4}}{r^{2}}-\frac{m \omega^{2}}{r^{2}}-\frac{1}{2} m \omega^{2} r^{2}\right] d t \mid \mathfrak{D} r\right. \\
= & -i\left(r^{\prime} r^{\prime \prime}\right)^{\frac{1}{2}} m \omega \csc (\omega \tau) \exp \left[\frac{1}{2} i m \omega\left(r^{\prime 2}+r^{\prime \prime 2}\right) \cot (\omega \tau)\right] \\
& \times I_{v}\left[-i m \omega r^{\prime} r^{\prime \prime} \csc (\omega \tau)\right],
\end{aligned}
$$

for $\operatorname{Re}(v)>-1$.

\section{The Charged Particle in a Uniform Magnetic Field}

The Lagrangian for a particle of charge $e$ moving in a constant uniform magnetic field $B$, which is applied along the $z$ axis, is

$$
L=\frac{1}{2} m\left[\dot{\mathbf{r}}^{2}+2 \omega(x \dot{y}-y \dot{x})\right]
$$

or, in cylindrical coordinates $(r, \theta, z)$,

$$
L=\frac{1}{2} m\left(\dot{r}^{2}+r^{2} \dot{\theta}^{2}+2 \omega r^{2} \dot{\theta}\right)+\frac{1}{2} m \dot{z}^{2},
$$

where $\frac{1}{2} e B / m=\omega$ is the Larmor frequency of the charged particle. Introduction of a new angular variable $\phi$ such that

$$
\phi=\theta+\omega t
$$

and

$$
\dot{\theta}^{2}+2 \omega \dot{\theta}=\dot{\phi}^{2}-\omega^{2}
$$

casts the Lagrangian (29) in the form

$$
L=\frac{1}{2} m\left(\dot{r}^{2}+r^{2} \dot{\phi}^{2}-\omega^{2} r^{2}\right)+\frac{1}{2} m \dot{z}^{2} .
$$

The corresponding partial action in the time interval $\epsilon$ is

$$
\begin{aligned}
S\left(\mathbf{r}_{j}, \mathbf{r}_{j-1}\right)= & \frac{1}{2} m\left(r_{j}^{2}+r_{j-1}^{2}\right) / \epsilon \\
& -(m / \epsilon) r_{j} r_{j-1} \cos \left(\phi_{j}-\phi_{j-1}\right) \\
& \quad-\frac{1}{2} \epsilon m \omega^{2} r_{j}^{2}+\frac{1}{2} m\left(\Delta z_{j}\right)^{2} / \epsilon .
\end{aligned}
$$

It is clear that the motion of a charged particle in a uniform magnetic field is equivalent to a combination of two-dimensional harmonic oscillation and free motion perpendicular to the plane of oscillation. Correspondingly, the action in a given time interval can be separated into contributions from the harmonic oscillation and the free motion. Thus, the propagator for this system is a product of the propagators for a harmonic oscillator in the $(r, \phi)$ plane and a free particle in the $z$ direction. That is,

$K\left(r^{\prime \prime}, \phi^{\prime \prime}, z^{\prime \prime} ; r^{\prime}, \phi^{\prime}, z^{\prime} ; \tau\right)$

$$
=K\left(r^{\prime \prime}, \phi^{\prime \prime} ; r^{\prime}, \phi^{\prime} ; \tau\right) K_{0}\left(z^{\prime \prime}, z^{\prime} ; \tau\right) .
$$

The propagators on the right-hand side of Eq. (62) have been expressed in Eqs. (53) and (55). Transforming the variable $\phi$ back into the real angular variable $\theta$ by Eq. (59) leads to the desired propagator

$$
\begin{aligned}
K\left(r^{\prime \prime}, \theta^{\prime \prime}, z^{\prime \prime} ; r^{\prime}, \theta^{\prime}, z^{\prime} ; \tau\right) & \\
= & \left(\frac{m}{2 \pi i}\right)^{\frac{3}{2}} \frac{\omega \tau}{\sin (\omega \tau)} \\
& \times \exp \left\{\frac { i m \omega } { 2 \operatorname { s i n } ( \omega \tau ) } \left[\left(r^{\prime 2}+r^{\prime 2}\right) \cos (\omega \tau)\right.\right. \\
& \left.\left.-2 r^{\prime} r^{\prime \prime} \cos \left(\theta^{\prime \prime}-\theta^{\prime}+\omega \tau\right)\right]+\frac{i m}{2 \tau}\left(z^{\prime \prime}-z^{\prime}\right)^{2}\right\}
\end{aligned}
$$

It is well known that the simple replacement of $\tau$ in the propagator by $-i(k T)^{-1}$, where $k$ is the Boltzman constant and $T$ the temperature, enables one to write down the density matrix in statistical mechanics. Following this procedure, we obtain the density matrix for an ensemble of charged particles 
in a uniform magnetic field as

$$
\begin{array}{r}
\rho\left(r^{\prime \prime}, r^{\prime} ; T\right)=\left(\frac{m k T}{2 \pi}\right)^{\frac{3}{2}} \frac{\omega k^{-1} T^{-1}}{\sin \left(\omega k^{-1} T^{-1}\right)} \exp \left\{-\frac{1}{2} m k T\right. \\
\times\left[\frac{2 i \omega}{k T} r^{\prime} r^{\prime \prime} \sin \left(\theta^{\prime}-\theta^{\prime \prime}\right)+\frac{\omega}{k T}\left(r^{\prime 2}+r^{\prime \prime 2}\right) \operatorname{coth}\left(\frac{\omega}{k T}\right)\right. \\
\left.\left.\quad-\frac{2 \omega}{k T} \operatorname{coth}\left(\frac{\omega}{k T}\right) \cos \left(\theta^{\prime \prime}-\theta^{\prime}\right)+\left(z^{\prime \prime}-z^{\prime}\right)^{2}\right]\right\}
\end{array}
$$

which is of the same form as that derived by Sondheimer and Wilson. ${ }^{5}$

\section{The Rigid Rotator}

The expression (33) for the $l$ wave is more convenient than (16) for evaluating the propagator of a rigid rotator. Let $r_{0}$ be the radius of the sphere on which the rotator is constrained. Then let $\delta\left(r_{j}-r_{0}\right)$ take the place of $\exp \left[-i \epsilon V\left(r_{j}\right)\right]$ in the radial propagator (33); that is,

$$
\begin{aligned}
K_{l}\left(r_{0} ; \tau\right)=r_{0}^{-2} & \lim _{N \rightarrow \infty}\left(\frac{m}{2 \pi i \epsilon}\right)^{\frac{1}{2}} \\
& \times \prod_{j=1}^{N}\left\{\operatorname { e x p } \left[\frac{i m}{2 \epsilon}\left(r_{j}-r_{j-1}\right)^{2}\right.\right. \\
& \left.\left.-\frac{i \epsilon l(l+1)}{r_{j} r_{j-1}}\right] \delta\left(r_{j}-r_{0}\right)\right\} \prod^{N-1}(d r) .
\end{aligned}
$$

After integration, the following simple form results:

$$
K_{l}\left(r_{0} ; \tau\right)=r_{0}^{-2} \exp \left[\frac{\tau}{2 i m} \frac{l(l+1)}{r_{0}}\right] .
$$

Thus Eq. (15) gives, for this rotator,

$$
\begin{aligned}
& K\left(\theta^{\prime \prime}, \phi^{\prime \prime} ; \theta^{\prime}, \phi^{\prime} ; \tau\right) \\
& \quad=r_{0}^{-1} \sum_{l=0}^{\infty} \sum_{n=-l}^{l} \exp \left[\frac{\tau l(l+1)}{2 i m r_{0}}\right] Y_{l}^{n^{*}}\left(\theta^{\prime \prime}, \phi^{\prime \prime}\right) Y_{l}^{n}\left(\theta^{\prime}, \phi^{\prime}\right) .
\end{aligned}
$$

The Particle in an Inverse-Square Potential

For a particle in an attractive potential

$$
V(r)=k^{2} / r^{2},
$$

the derivation of the propagator is a trivial matter when one utilizes relation (56), setting $\omega=0$ and replacing $l+\frac{1}{2}$ by $\left[\left(l+\frac{1}{2}\right)^{2}+k^{2}\right]^{\frac{1}{2}}$. To see the situation in more detail, we start with the radial function (31), which now takes the form

$$
\begin{aligned}
& R_{l}\left(r_{j}, r_{j-1}\right)=\frac{i \epsilon}{2 m r_{j} r_{j-1}} \\
& \times \exp \left\{\frac{i m}{2 \epsilon}\left(\Delta r_{j}\right)^{2}-\frac{i \epsilon\left[l(l+1)+k^{2}\right]}{2 m r_{j} r_{j-1}}\right\} .
\end{aligned}
$$

Within the approximation adopted, the asymptotic expansion formula (22) enables us to rewrite the radial function as

$$
\begin{aligned}
R_{l}\left(r_{j}, r_{j-1}\right) & =\left(\frac{i \pi \epsilon}{2 m r_{j} r_{j-1}}\right)^{\frac{1}{2}} \\
& \times \exp \left[\frac{i m\left(r_{j}^{2}+r_{j-1}^{2}\right)}{2 \epsilon}\right] I_{\lambda}\left(\frac{m r_{j} r_{j-1}}{i \epsilon}\right),
\end{aligned}
$$

where

$$
\lambda(l)=\left[\left(l+\frac{1}{2}\right)^{2}+k^{2}\right]^{\frac{1}{2}} .
$$

Since the radial integrations are independent of $\lambda(l)$, there results from Eq. (70) in much the same manner that Eq. (33) comes from Eq. (31) the radial propagator for the $l$ wave

$$
\begin{aligned}
& K_{l}\left(r^{\prime \prime}, r^{\prime} ; \tau\right)=\left(r^{\prime} r^{\prime \prime}\right)^{\frac{1}{2}}(-i m / \tau) \\
& \quad \times \exp \left[\frac{1}{2} i m\left(r^{\prime 2}+r^{\prime \prime 2}\right) / \tau\right] I_{\lambda}\left(-i m r^{\prime} r^{\prime \prime} / \tau\right),
\end{aligned}
$$

with $\lambda$ defined by (71). By Eq. (15), the propagator for a particle in the potential (68) is

$$
\begin{aligned}
& K\left(r^{\prime \prime}, \theta^{\prime \prime}, \phi^{\prime \prime} ; r^{\prime}, \theta^{\prime}, \phi^{\prime} ; \tau\right) \\
& =\left(r^{\prime} r^{\prime \prime}\right)^{-\frac{1}{2}}\left(\frac{m}{i \tau}\right) \exp \left[\frac{i m}{2 \tau}\left(r^{\prime 2}+r^{\prime \prime 2}\right)\right] \\
& \quad \times \sum_{l=0}^{\infty} \sum_{n=-l}^{l} I_{\lambda(l)}\left(\frac{m r^{\prime} r^{\prime \prime}}{i \tau}\right) Y_{l}^{n^{*}}\left(\theta^{\prime \prime}, \phi^{\prime \prime}\right) Y_{l}^{n}\left(\theta^{\prime}, \phi^{\prime}\right) .
\end{aligned}
$$

\section{ACKNOWLEDGMENT}

The authors wish to acknowledge helpful discussions with Professor Jack H. Smith.

\section{APPENDIX A: DERIVATION OF FORMULA (41)}

Consider the contour integral

$$
\oint_{\Gamma} e^{-\alpha z^{2}} I_{v}(a z) I_{v}(b z) z d z
$$

for $\operatorname{Re}(v)>-1$ and $\operatorname{Re}(\alpha)>0$. As is shown in Fig. 1, $\Gamma$ is a closed contour consisting of a path from $A$ to $B$ along the positive real axis, a circular arclike path from $B$ to $C$, a path from $C$ to $D$ along the line with $\arg (z)=3 \pi / 4$, and a small circular arclike path from $D$ to $A$ about the origin. The integrand is regular in the $z$ plane cut along the negative real axis. As a consequence, the integral (A1) vanishes. Since the contributions from the two arclike paths disappear when the appropriate limits are invoked, we have

$$
\begin{aligned}
i \int_{0}^{\infty} e^{i \alpha z^{2}} I_{v}\left(a z / i^{\frac{1}{2}}\right) I_{v}\left(b z / i^{\frac{1}{2}}\right) z d z \\
\\
+\int_{0}^{\infty} e^{-\alpha z^{2}} I_{v}(z a) I_{v}(b z) z d z=0 .
\end{aligned}
$$




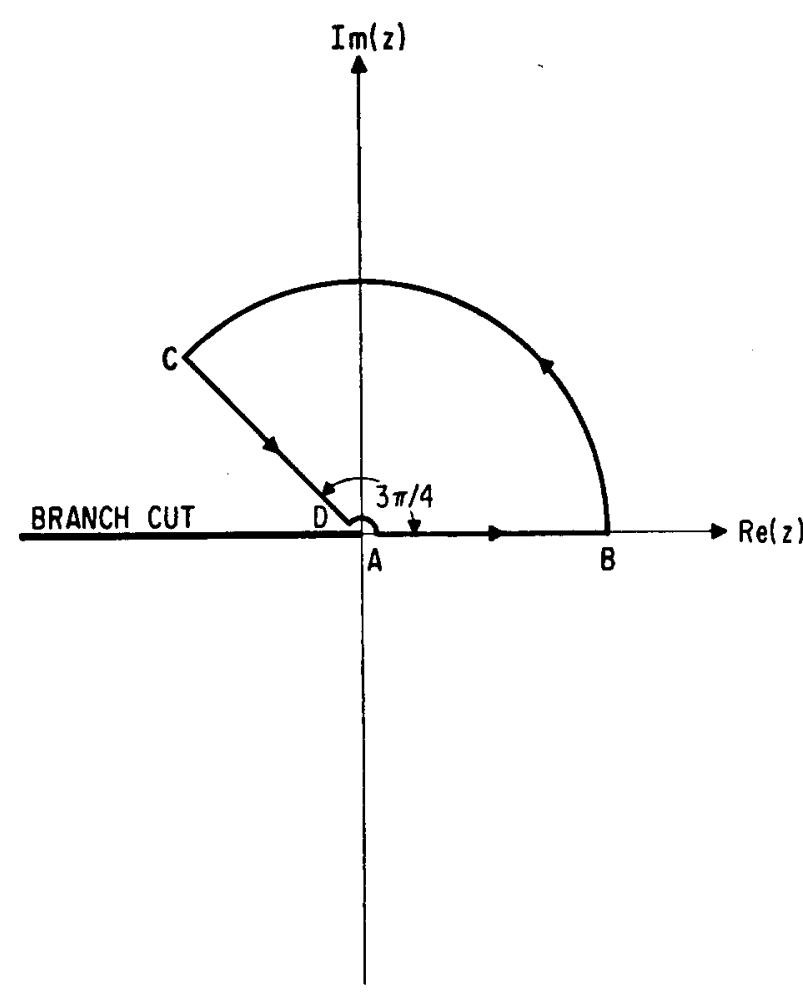

FIG. 1. Contour $\Gamma$ taken in Eq. (A1).

Thus, by Weber's formula?

$$
\int_{0}^{\infty} e^{-\alpha z^{2}} I_{v}(a z) I_{v}(b z) z d z=\frac{1}{2 \alpha} \exp \left[\frac{a^{2}+b^{2}}{4 \alpha}\right] I_{v}\left(\frac{a b}{2 \alpha}\right),
$$

we obtain our formula (41), after replacing $a$ by $a / i^{\frac{1}{2}}$ and $b$ by $b / i^{\frac{1}{2}}$.

\section{APPENDIX B: DETERMINATION OF THE COEFFICIENTS $a, f$, AND $g$}

Let $\lambda_{j}$ be $2 \alpha_{j} / \beta$ and define the finite product of $\lambda_{j}^{-1}$ :

$$
\Lambda_{k}=\prod_{j=1}^{k} \lambda_{j}^{-1}
$$

Then the coefficients defined in Eqs. (46), (47), and (48) are all expressible in terms of $\beta$ and $\Lambda_{k}$ :

$$
\begin{aligned}
& a_{N}=\beta \Lambda_{N-1}, \\
& f_{N}=\frac{1}{2} \beta\left(1-\sum_{j=1}^{N-1} \Lambda_{j} \Lambda_{j-1}\right), \\
& g_{N}=\frac{1}{2} \beta\left(1-\Lambda_{N}^{\cdot} / \Lambda_{N-1}\right) .
\end{aligned}
$$

${ }^{7}$ See G. N. Watson, A Treatise on the Theory of Bessel Functions (Cambridge University Press. Cambridge, England, 1962), 2nd ed., p.395.
Now consider a series

$$
X_{k}=\sum_{j=0}^{k}(-1)^{j}\left(\begin{array}{c}
k+j+1 \\
2 j+1
\end{array}\right) \eta^{2 j+1}
$$

By induction, it is straightforward to show that

$$
X_{k+1}+X_{k-1}=X_{1} X_{k} \text {. }
$$

It is apparent that

$$
\lambda_{k}=X_{k} / X_{k-1}
$$

satisfies the relation

$$
\lambda_{k+1}+\lambda_{k}^{-1}=\lambda_{1},
$$

which coincides with Eq. (43) for $\lambda_{j}=2 \alpha_{j} / \beta$. From (B7) it immediately follows that

$$
\Lambda_{k}=X_{1} / X_{k},
$$

where $\eta$ is $\omega \epsilon$.

Let $N$ and $\eta$ be such that $N \eta$ remains finite for all $N$. Then

$$
X_{k-1} \rightarrow \sin (k \eta)
$$

as $N$ goes to infinity. To see this, compare the sum of the first $n$ terms of $X_{k-1}$,

$$
T_{n}=\sum_{j=0}^{n<k}(-1)^{j}\left(\begin{array}{c}
k+j \\
2 j+1
\end{array}\right) \eta^{2 j+1}
$$

with that of the series for $\sin (k \eta)$,

$$
S_{n}=\sum_{j=0}^{n<k}(-1)^{j} \frac{(k \eta)^{2 j+1}}{(2 j+1) !}
$$

that is,

$$
\left|T_{n}-S_{n}\right|<[n(n+1) \eta / k] \sinh (k \eta),
$$

from which the convergence (B10) is obvious.

Accordingly, we have

$$
\begin{aligned}
& a_{N} \rightarrow \beta \eta \csc (N \eta), \\
& f_{N} \rightarrow \frac{1}{2} \beta \eta \cot (N \eta) .
\end{aligned}
$$

It is also clear that $\left(\Lambda_{k} \Lambda_{k-1}\right)$ converges uniformly to $\eta^{2} \csc [(k+1) \eta] \csc (k \eta)$ in the same limit. Therefore, we may write

$$
\lim _{N \rightarrow \infty} \sum_{j=1}^{N-1}\left(\Lambda_{k} \Lambda_{k-1}\right)^{-1}=\eta \int_{\eta}^{N \eta} \csc ^{2} x d x
$$

and determine the limiting value of $g$ as

$$
g_{N} \rightarrow \frac{1}{2} \beta \eta \cot (N \eta) .
$$

In Eqs. (B14), (B15), and (B17), let $\beta \eta=m \omega$ and $N \eta=\omega \tau$. 